\title{
Projected trust region method for stochastic linear complementarity problems
}

\author{
Ying-xiao Wang, Shou-qiang Du*, Huan-huan Li, Miao Chen \\ School of Mathematics and Statistics, Qingdao University, Qingdao, Shandong, China 266071
}

*Corresponding author, e-mail: sqdu@qdu.edu.cn

Received 11 Dec 2017

Accepted 12 Oct 2018

\begin{abstract}
In this paper, we consider the method for solving a kind of general stochastic linear complementarity problems. Based on Fischer-Burmeister function, we transform this kind of general stochastic linear complementarity problems into nonsmooth equations and present a projected trust region method to solve the nonsmooth equations. The global convergence of this method is also given. Finally, the numerical results are reported.
\end{abstract}

KEYWORDS: nonsmooth equations, global convergence

MSC2010: 90C33 65K10 90C15

\section{INTRODUCTION}

With the continuous maturity and development of financial markets in various countries, the trading system and tools in the financial field are becoming more and more perfect. The types and forms of representative options in financial derivatives are constantly and increasingly developed. An option in a derivative is the right to buy and sell assets at a certain price. In the field of finance and mathematics, the pricing of derivative securities, represented by option pricing, has been studied a long time ${ }^{1,2}$. Because American option is more complex than European option, the current American option pricing problem is still one of the most important problems in the derivative securities pricing problem. In the case of no arbitrage, the equation was converted to a stochastic complementarity problem ${ }^{3}$, which is based on the definition of the portfolio. The portfolio is constructed when the Black-Scholes equation is derived. Besides, the prices of American options are depended on the asset price, the strike price, the expiration date, the risk-free rate and the volatility of the asset price, which is usually assumed as constant. However, since each expert has its own views on volatility, it is unsuitable to set up the volatility as a constant ${ }^{4}$. Ref. 5 provided the relationship of option pricing and linear complementarity problems and Ref. 4 proposed a kind of stochastic linear complementarity problems to solve the problem. They also gave the existence conditions of the solution. On the other hand, the stochastic linear complementarity problems has an important application in engineering ${ }^{6}$, transportation ${ }^{7}$ and others ${ }^{8}$. For example, the refinery production and the demand depend on the output of oil and the weather ${ }^{8}$, respectively, which change every day with uncertainty. The refinery production problem can be transformed into a stochastic linear complementarity problem with four random variations. This shows that the stochastic linear complementarity problems is one of the important mathematical models in the financial problems. Hence in this study, we present a more general stochastic linear complementarity problem, which contains the models of the above problems.

Assume that $(\Omega, F, P)$ is a probability space with $\Omega \subseteq \mathbb{R}^{n}$, where the probability distribution $P$ is known. A general stochastic linear complementarity problem is to find a vector $x \in \mathbb{R}^{n}$ such that

$$
\begin{array}{r}
F(x, \omega)=\left(A_{1}(\omega)+\lambda I\right) x-b_{1}(\omega) \geqslant 0, \\
G(x, \omega)=\left(A_{2}(\omega)-\lambda I\right) x-b_{2}(\omega) \geqslant 0, \\
F(x, \omega)^{\mathrm{T}} G(x, \omega)=0,
\end{array}
$$

where $\Omega \subseteq \mathbb{R}^{n}$ is the underlying sample space and $\omega \in \Omega=\left\{\omega_{1}, \omega_{2}, \ldots, \omega_{n}\right\}$ is a random vector with given probability distribution $P, \lambda \in \mathbb{R}$ and $I$ is $n \times n$ identity matrix. For each $\omega, A_{1}(\omega), A_{2}(\omega), b_{1}(\omega)$, and $b_{2}(\omega)$ are $n \times n$ matrices and $n$-dimensional vectors, respectively. When $\lambda=0$, the above problem is transformed into the general stochastic linear complementarity problems ${ }^{9}$, i.e., to find a vector 
$x \in \mathbb{R}^{n}$ such that

$$
\begin{aligned}
F(x, \omega)= & M_{1}(\omega) x+q_{1}(\omega) \geqslant 0, \\
G(x, \omega)= & M_{2}(\omega) x+q_{2}(\omega) \geqslant 0, \\
& F(x, \omega)^{\mathrm{T}} G(x, \omega)=0 .
\end{aligned}
$$

When $F(x, \omega)=x$, the above problem is transformed into the stochastic linear complementarity problems ${ }^{8,10}$. This kind of problems is usually solved by expectancy value (EV) method and expected residue minimization method, which are widely studied in many articles ${ }^{11,12}$. When $\Omega$ includes only one element, problem (1) is transformed into the general linear complementarity problems ${ }^{13}$, i.e., to find a vector $x \in \mathbb{R}^{n}$ such that

$$
\begin{array}{r}
F(x)=M_{1}(x)+q_{1} \geqslant 0, \\
G(x)=M_{2}(x)+q_{2} \geqslant 0, \\
F(x)^{\mathrm{T}} G(x)=0,
\end{array}
$$

where $M_{1}$ and $M_{2}$ are $n \times n$ matrices, $q_{1}$ and $q_{2}$ are $n$-dimensional vectors.

When $G(x, \omega)=x$, the general linear complementarity problems are transformed into the linear complementarity problems (LCP), which are widely used in solving absolute value equations, equilibrium problems and the related problems. For more details of the applications of LCP see Refs. 14, 15. The interested readers can also refer to two monographs (Refs. 16,17) by Cottle et al.

There are many well-known methods for solving LCP and we consider some methods to solve problem (1). To transform the problem (1) to LCP, we use the EV method to obtain the following equations. Denote

$$
\begin{aligned}
\overline{A_{1}+\lambda I} & =\sum_{i=1}^{m} p_{i}\left(A_{1}\left(\omega_{i}\right)+\lambda I\right), \\
\overline{b_{1}} & =\sum_{i=1}^{m} p_{i} b_{1}\left(\omega_{i}\right),
\end{aligned}
$$

where $p_{i}=P\left(\omega_{i} \in \Omega\right) \geqslant 0, i=1, \ldots, m$. The problem (1) is equivalent to the problem defined as

$$
\begin{gathered}
\overline{\left(A_{1}+\lambda I\right)} x-\overline{b_{1}} \geqslant 0, \overline{\left(A_{2}-\lambda I\right)} x-\overline{b_{2}} \geqslant 0, \\
\left(\overline{\left(A_{1}+\lambda I\right)} x-\overline{b_{1}}\right)^{\mathrm{T}}\left(\overline{\left(A_{2}-\lambda I\right)} x-\overline{b_{2}}\right)=0,
\end{gathered}
$$

and

$$
\begin{aligned}
& \left(A_{1}\left(\omega_{i}\right)+\lambda I\right) x-b_{1}\left(\omega_{i}\right) \geqslant 0, \\
& \left(A_{2}\left(\omega_{i}\right)-\lambda I\right) x-b_{2}\left(\omega_{i}\right) \geqslant 0,
\end{aligned}
$$

for $i=1, \ldots, m$. The problem (4) is a general linear complementarity problem, so we can use the complementarity functions to solve this problem. The complementarity functions have many different forms, such as the functions proposed in Refs. 18, 19. Among them, Fischer-Burmeister function $^{20} \phi(a, b)=\sqrt{a^{2}+b^{2}}-(a+b)$ is convex ${ }^{13}$ and differentiable at any point $(a, b) \neq(0,0)$. Furthermore, the square of the Fischer-Burmeister function is continuously differentiable at any point in the plane. Hence we use the Fischer-Burmeister function to transform problem (4) and solving problem (4) is equivalent to solve $\Phi(x)=0$, where

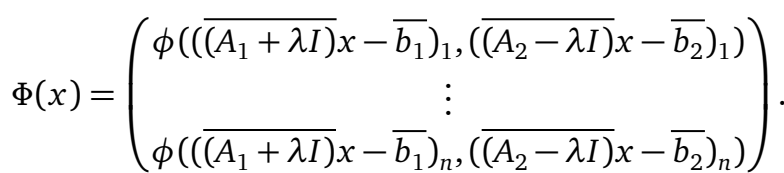

To solve problem (4) and problem (5), it is equivalent to solve the following problem,

$$
H(x, y)=0, \quad y \geqslant 0,
$$

where

$$
\begin{gathered}
H(x, y)=\left(\begin{array}{c}
\left(A_{1}\left(\omega_{1}\right)+\lambda I\right) x-b_{1}\left(\omega_{1}\right)-y_{1} \\
\vdots \\
\left(A_{1}\left(\omega_{m}\right)+\lambda I\right) x-b_{1}\left(\omega_{m}\right)-y_{m} \\
\left(A_{2}\left(\omega_{1}\right)-\lambda I\right) x-b_{2}\left(\omega_{1}\right)-y_{m+1} \\
\vdots \\
\left(A_{2}\left(\omega_{m}\right)+\lambda I\right) x-b_{2}\left(\omega_{m}\right)-y_{2 m}
\end{array}\right), \\
y=\left(y_{1}^{\mathrm{T}}, y_{2}^{\mathrm{T}}, \cdots, y_{2 m}^{\mathrm{T}}\right)^{\mathrm{T}} \in \mathbb{R}^{2 m \times n}
\end{gathered}
$$

For $x=x^{\prime}-x^{\prime \prime}$, where $x^{\prime}, x^{\prime \prime} \in \mathbb{R}^{n}$, and $x^{\prime}, x^{\prime \prime} \geqslant 0$, let $z=\left(x^{\prime}, x^{\prime \prime}, y\right) \in \mathbb{R}^{(2 m+2) n}$ and define a merit function of (6) as $f(z)=\frac{1}{2}\|H(z)\|^{2}$. If problem (1) has a solution, then solving (6) is equivalent to find a global solution of the optimization problem,

$$
\min _{z \in \Omega_{1}} f(z),
$$

where $\Omega_{1}=\{z \mid z \geqslant 0\}$.

To make the transformation of problem (1) more distinct, we give a concrete example to explain the transformation process. Consider problem (1), where

$$
\begin{aligned}
& A_{1}(\omega)=\left(\begin{array}{cc}
1+\omega & 1 \\
1 & 2
\end{array}\right), \quad b_{1}(\omega)=\left(\begin{array}{c}
1+\omega \\
1
\end{array}\right), \\
& A_{2}(\omega)=\left(\begin{array}{cc}
1+\omega & 1 \\
1 & 1+\omega
\end{array}\right), \quad b_{2}(\omega)=\left(\begin{array}{c}
\omega \\
1+\omega
\end{array}\right),
\end{aligned}
$$

$\Omega=\{0,1\}, p_{i}=P\left(\omega_{i} \in \Omega\right)=0.5, i=1,2$, and $\lambda=1$.

Let

$$
\begin{array}{r}
F(x, \omega)=\left(A_{1}(\omega)+I\right) x-b_{1}(\omega) \geqslant 0, \\
G(x, \omega)=\left(A_{2}(\omega)-I\right) x-b_{2}(\omega) \geqslant 0, \\
F(x, \omega)^{\mathrm{T}} G(x, \omega)=0 .
\end{array}
$$


Then

$$
\begin{aligned}
\overline{A_{1}+I} & =\sum_{i=1}^{2} p_{i}\left(A_{1}\left(\omega_{i}\right)+I\right)=\left(\begin{array}{ll}
\frac{5}{2} & 1 \\
1 & 3
\end{array}\right), \\
\overline{b_{1}} & =\sum_{i=1}^{2} p_{i} b_{1}\left(\omega_{i}\right)=\left(\begin{array}{c}
\frac{3}{2} \\
1
\end{array}\right), \\
\overline{A_{2}-I} & =\sum_{i=1}^{2} p_{i}\left(A_{2}\left(\omega_{i}\right)-I\right)=\left(\begin{array}{ll}
\frac{1}{2} & 1 \\
1 & \frac{1}{2}
\end{array}\right), \\
\overline{b_{2}} & =\sum_{i=1}^{2} p_{i} b_{2}\left(\omega_{i}\right)=\left(\begin{array}{l}
\frac{1}{2} \\
\frac{3}{2}
\end{array}\right) .
\end{aligned}
$$

We obtain

$\Phi(x)=\left(\begin{array}{l}\phi\left(\left[\overline{\left(A_{1}+\lambda I\right)} x-\overline{b_{1}}\right]_{1},\left[\overline{\left(A_{2}-\lambda I\right)} x-\overline{b_{2}}\right]_{1}\right) \\ \phi\left(\left[\overline{\left(A_{1}+\lambda I\right)} x-\overline{b_{1}}\right]_{2},\left[\overline{\left(A_{2}-\lambda I\right)} x-\overline{b_{2}}\right]_{2}\right)\end{array}\right)$

and

$$
H(z)=H(x, y)=\left(\begin{array}{c}
\Phi(x) \\
2 x_{1}+x_{2}-1-y_{11} \\
x_{1}+3 x_{2}-1-y_{12} \\
3 x_{1}+x_{2}-2-y_{21} \\
x_{1}+3 x_{2}-1-y_{22} \\
x_{2}-y_{31} \\
x_{1}-1-y_{32} \\
x_{1}+x_{2}-1-y_{41} \\
x_{1}+x_{2}-2-y_{42}
\end{array}\right)=0
$$

with $y=\left(y_{11}, y_{12}, y_{21}, y_{22}, y_{31}, y_{32}, y_{41}, y_{42}\right) \geqslant 0$. The problem (9) is transformed into a nonnegative constrained optimization problem.

There are many methods to solve problem (8) and the related optimization problems, such as Refs. 21, 22. The Levenberg-Marquardt method is an important method that has been widely used in recent years ${ }^{23,24}$. It overcomes the fact that the Gauss-Newton method requires full rank of the Jacobian matrix's column of $H$ in the iteration process. And the convergence rate of Levenberg-Marquardt method is quadratic. However, the descent direction of the Levenberg-Marquardt method is

$$
d_{k}=-\left[V_{k}^{\mathrm{T}} V_{k}+\mu_{k} I\right]^{-1} V_{k}^{\mathrm{T}} H\left(z_{k}\right),
$$

where $V_{k} \in \partial H\left(z_{k}\right)$ and $\mu_{k}$ is Lagrange multiplier. $d_{k}$ is related to the value of $\mu_{k}$ and it is difficult to define a suitable value of $\mu_{k}$. Fortunately, trust region method is a method which is related closely to the Levenberg-Marquardt method. Furthermore, trust region method could adjust the radius by quadratic model to obtain a more sufficient descent of the objective function. The positivity of the Hessian matrix is not necessary in the projected trust region method. Hence the projected trust region method is also widely used ${ }^{25,26}$. In this work the projected trust region method is used to solve for the special structure of problem (1).

\section{PROJECTED TRUST REGION METHOD}

This section provides some preliminaries ${ }^{17,27}$ and proposes the projected trust region method for solving the given general stochastic linear complementarity problems.

Definition 1 For a local Lipschitzian function $F$ : $\mathbb{R}^{m} \rightarrow \mathbb{R}^{n}$, the $B$-subdifferential of $F$ at $z$ is

$$
\partial_{B} F(z)=\left\{V \in \mathbb{R}^{m \times n} \mid \exists\left\{z_{k}\right\} \subseteq D_{F}:\left\{z_{k}\right\} \rightarrow z, F^{\prime}\left(z_{k}\right) \rightarrow V\right\}
$$

where $D_{F}$ is the set of differentiable points and $F^{\prime}\left(z_{k}\right)$ is the Jacobian of $F$ at $z_{k} \in \mathbb{R}^{n}$.

Clarke's generalized Jacobian of $F$ at $z \in \mathbb{R}^{n}$ is

$$
\partial_{\mathrm{C}} F(z)=\operatorname{conv}\left\{\partial_{B} F(z)\right\}
$$

where conv denotes the convex hull of a set. Note that

$$
\partial_{\mathrm{C}} F(z)^{\mathrm{T}}=\partial F_{1}(z) \times \cdots \times \partial F_{n}(z) .
$$

For any $z=\left(x^{\prime}, x^{\prime \prime}, y\right) \in \mathbb{R}^{(2 m+2) n}$, we have

$$
\begin{aligned}
& \partial_{\mathrm{C}} H\left(x^{\prime}, x^{\prime \prime}, y\right) \\
& =\left(\begin{array}{ccccc}
V_{\phi} & -V_{\phi} & 0 & \cdots & 0 \\
A\left(\omega_{1}\right)+\lambda I & -\left(A\left(\omega_{1}\right)+\lambda I\right) & -I & \cdots & 0 \\
\vdots & \vdots & \vdots & \vdots & \vdots \\
A\left(\omega_{m}\right)+\lambda I & -\left(A\left(\omega_{m}\right)+\lambda I\right) & 0 & \cdots & 0 \\
A\left(\omega_{1}\right)-\lambda I & -\left(A\left(\omega_{1}\right)-\lambda I\right) & 0 & \cdots & 0 \\
\vdots & \vdots & \vdots & \vdots & \vdots \\
A\left(\omega_{1}\right)-\lambda I & -\left(A\left(\omega_{1}\right)-\lambda I\right) & 0 & \cdots & -I
\end{array}\right)
\end{aligned}
$$

where $V_{\phi} \in \partial_{C} \phi(x), I$ is the $n \times n$ identity matrix, and $\lambda \in \mathbb{R}$.

Definition $2 F$ is said to be semi-smooth at $z$ if

$$
\lim _{\substack{V \in \partial_{\mathrm{C}} F\left(z+t h^{\prime}\right) \\ h^{\prime} \rightarrow h, t \downarrow 0}} V h^{\prime}
$$

exists for any $h \in \mathbb{R}^{n}$.

Definition $3 F$ is said to be strongly semi-smooth at $z$ if $F$ is semi-smooth at $z$ and

$$
\lim _{\substack{V \in \partial_{C} F(z+h) \\ h \rightarrow 0}} \frac{\|F(z+h)-F(z)-V h\|}{\|h\|^{2}}<\infty .
$$


The projected trust region method contains elements from affine-scaling methods. By the methods proposed $^{28,29}$, the first-order optimization condition of problem (8) is equivalent to the nonlinear system

$$
\psi(z)=D(z) \nabla f(z)=0,
$$

where

$$
D(z)=\operatorname{diag}\left(d_{1}(z), \ldots, d_{n}(z)\right)
$$

is a suitable scaling matrix for $z \in \Omega_{1}$ that satisfies conditions

$$
d_{i}(z)\left\{\begin{array}{l}
=0, \quad z_{i}=0, \quad[\nabla f(z)]_{i}>0, \\
=0, \quad z_{i}=\infty, \quad[\nabla f(z)]_{i}<0, \\
\geqslant 0, \quad z_{i} \geqslant 0, \quad[\nabla f(z)]_{i}=0, \\
>0, \quad \text { otherwise, }
\end{array}\right.
$$

for $i=1, \ldots, n$. Some examples of $D(z)$ are given in Refs. 29,30. In this study, the form of $D(z)$ is defined based on Ref. 29, namely,

$$
d_{i}(z)= \begin{cases}\min \left\{1, z_{i}\right\}, & {[\nabla f(z)]_{i} \geqslant 0,} \\ 1, & {[\nabla f(z)]_{i}<0,}\end{cases}
$$

for $i=1, \ldots, n$ and $z \in \Omega_{1}$.

To solve problem (8), let $z_{k}$ be the $k$ th iteration point, denote $f_{k}=f\left(z_{k}\right), g_{k}=\nabla f\left(z_{k}\right)=H\left(z_{k}\right)^{\mathrm{T}} V_{k}$, and $V_{k} \in \partial_{\mathrm{C}} H\left(z_{k}\right)$, and define $B_{k}=V_{k}^{\mathrm{T}} V_{k}+\mu_{k} I$, where $\mu_{k}=\mu\left\|f\left(z_{k}\right)\right\|^{2}, \mu \in(0,1)$. The trust region subproblem of $k$ th iteration has the form

$$
\min _{\substack{\|p\|_{\infty} \leqslant \Delta_{k} \\ p+z_{k} \geqslant 0}} q_{k}(p)=g_{k}^{\mathrm{T}} p+\frac{1}{2} p^{\mathrm{T}} B_{k} p,
$$

where $\Delta_{k}$ is the radius of projected trust region and $z_{k}$ is the mean of $k$ th iteration. Suppose that the optimum solution of problem (10) is $d_{k}$, the actual decrease of $f$ in the $k$ th step is

$$
\Delta f_{k}=f\left(z_{k}\right)-f\left(z_{k}+d_{k}\right)
$$

and the predicted decrease is

$$
\Delta q_{k}=q_{k}(0)-q_{k}\left(d_{k}\right)
$$

with the ratio $r_{k}=\Delta f_{k} / \Delta q_{k}$. The solution $p_{k}$ of problem (10) satisfies the Cauchy decrease condition

$$
q_{k}\left(p_{k}\right) \leqslant \alpha q_{k}\left(p_{k}^{c}\right)
$$

where $\alpha \in(0,1)$ and $p_{k}^{c}=p\left(t_{k}\right)$ is the scaled Cauchy step, when $t_{k}$ is a solution of

$$
\min _{\substack{p(t)=-t D_{k}^{2} g_{k}, t \geqslant 0 \\\|p(t)\| \leqslant \Delta_{k}, z_{k}+p(t) \geqslant 0}} q_{k}(p(t)) .
$$

Proposition 1 (Ref. 30) The function $f(z)=$ $\frac{1}{2}\|H(z)\|^{2}$ defined in (7) is continuously differentiable with its gradient $V_{k}^{\mathrm{T}} H(z)$, where $V_{k} \in \partial H(z)$.

From Refs. 29,31, the projected trust region method for solving problem (1) is described as follows.

\section{Method 1. The projected trust region method}

Choose $0<\eta_{1}<\eta_{2}<1,0<\gamma_{1}<1<\gamma_{2}, \Delta_{\max }>0$, initial radius of trust region $\Delta_{0}<\Delta_{\text {max }}$, initial point $z_{0}$, parameters $\mu, \rho, \epsilon \in(0,1)$, and set $k=0$.

Step 1: Compute $g_{k}=\nabla f\left(z_{k}\right)$. If $\left\|D_{k} g_{k}\right\|<\epsilon$, stop. Step 2: Choose $V_{k} \in \partial H\left(z_{k}\right)$, compute $d_{k}^{\mathrm{LM}}$ by

$$
\left(V_{k}^{\mathrm{T}} V_{k}+\mu_{k} I\right) d_{k}^{\mathrm{LM}}=-V_{k}^{\mathrm{T}} H\left(z_{k}\right) .
$$

Step 3: If $\left\|f\left(P_{\Omega_{1}}\left(z_{k}\right)\right)\right\| \leqslant \rho\left\|f\left(z_{k}\right)\right\|$ holds, set

$$
z_{k+1}=P_{\Omega_{1}}\left(z_{k}\right), \quad \Delta_{k+1}=\min \left(\gamma_{2} \Delta_{k}, \Delta_{\max }\right),
$$

and go to Step 7. Otherwise, go to Step 4 .

Step 4: Solve subproblem (10) to obtain the solution $p_{k}$ and compute $\operatorname{Pred}\left(p_{k}\right)=\Delta q_{k}$, $\operatorname{Ared}\left(p_{k}\right)=\Delta f_{k}$, and $r_{k}$.

Step 5: Correct trust region radius

$$
\Delta_{k+1}= \begin{cases}\gamma_{1} \Delta_{k}, & r_{k} \leqslant \eta_{1}, \\ \Delta_{k}, & \eta_{1}<r_{k}<\eta_{2}, \\ \min \left\{\gamma_{2} \Delta_{k}, \Delta_{\max }\right\}, & \text { otherwise. }\end{cases}
$$

Step 6: If $r_{k}>\eta_{1}$, set $z_{k+1}=z_{k}+p_{k}$. Otherwise, set $z_{k+1}=z_{k}$.

Step 7: Set $k=k+1$ and go to Step 1 .

\section{CONVERGENCE ANALYSIS}

This section provides the global and local convergence properties of the projected trust region method based on the following lemmas proposed in Ref. 29.

Lemma 1 (Ref. 29) Let $p_{k}$ be a solution of the subproblem (10) satisfying (11). Then

$$
q_{k}(0)-q_{k}\left(p_{k}^{c}\right) \geqslant \frac{1}{2} \alpha\left\|D_{k} g_{k}\right\| \min \left\{\Delta_{k}, 1, \frac{\left\|g_{k}\right\|}{\left\|B_{k}\right\|}\right\} .
$$

Proof: From the definition of $V_{k}, V_{k}^{\mathrm{T}} V_{k}$ is positive semidefinite. Thus $V_{k}^{\mathrm{T}} V_{k}+\mu_{k} I$ is positive definite. Then the result is similar to the proof of Lemma 1 in Ref. 25.

Lemma 2 (Ref. 29) If $\left\{d_{k}^{\text {PLM }} \mid d_{k}^{\text {PLM }}=P_{\Omega_{1}}\left(z_{k}+\right.\right.$ $\left.\left.d_{k}^{\mathrm{LM}}\right)-z_{k}\right\}$ is infinite, then $\lim _{k \rightarrow \infty}\left\|H\left(z_{k}\right)\right\|=0$. 
Assumption 1 For the initial point $z_{0}, f(z)$ has a lower bound and is continuously differentiable on the level set $L\left(z_{0}\right)=\left\{z \mid f(z) \leqslant f\left(z_{0}\right)\right\}$.

Theorem 1 Suppose Assumption 1 holds. Let $\left\{z_{k}\right\}$ be a sequence generated by Method 1, and $z^{*}$ is an accumulation point of $\left\{z_{k}\right\}$. If Method 1 does not terminate in a finite number of steps, then

$$
\liminf _{k \rightarrow \infty}\left\|\nabla f\left(z_{k}\right)\right\|=0 .
$$

Proof: Without loss of generality, we assume that $\left\{z_{k}\right\}$ is an infinite sequence. By Lemma 2, the global convergence properties is not destroyed by $d_{k}^{\text {PLM }}$, the projected Levenberg-Marquardt method. Suppose that the descent direction is obtained by Step 4 and obtain $\nabla f\left(z_{k}\right) \neq 0$. To prove by contradiction, we assume that $\liminf _{k \rightarrow \infty} \nabla f\left(z_{k}\right) \neq 0$. This gives

$$
\begin{gathered}
\left|r_{k}-1\right|=\left|\frac{f\left(z_{k}\right)-f\left(z_{k}+d_{k}\right)-q_{k}(0)+q_{k}\left(d_{k}\right)}{q_{k}(0)-q_{k}\left(d_{k}\right)}\right| \\
=\left|\frac{f\left(z_{k}\right)-f\left(z_{k}+d_{k}\right)+q_{k}\left(d_{k}\right)}{q_{k}(0)-q_{k}\left(d_{k}\right)}\right| .
\end{gathered}
$$

From Taylor expansion,

$$
f\left(z_{k}+d_{k}\right)=f\left(z_{k}\right)+g\left(z_{k}\right)^{\mathrm{T}} d_{k}+o\left(d_{k}\right) .
$$

Since $V_{k}$ is upper semicontinuous ${ }^{32}, \partial_{B} H\left(z_{k}\right)$ is nonempty compact set at any point and bounded in bounded set of points. Thus $\partial_{\mathrm{C}} H\left(z_{k}\right)$ is bounded. Since $V_{k}$ is bounded for any $V_{k} \in \partial_{C} H\left(z_{k}\right), B_{k}=$ $V_{k}^{\mathrm{T}} V_{k}+\mu_{k} I$ is bounded and there exists a positive constant $c$ such that

$$
\left\|B_{k}\right\|=\left\|V_{k}^{\mathrm{T}} V_{k}+\mu_{k} I\right\| \leqslant c .
$$

This gives

$$
\begin{aligned}
\mid f\left(z_{k}\right)-f\left(z_{k}+d_{k}\right) & \left.+q_{k}\left(d_{k}\right)\right)|=| \frac{1}{2} d_{k}^{\mathrm{T}} B_{k} d_{k}-o\left(d_{k}\right) \mid \\
& \leqslant \frac{1}{2} c\left\|d_{k}\right\|^{2}+o\left(\left\|d_{k}\right\|\right) .
\end{aligned}
$$

From Ref. 29, there exists a positive constant $\epsilon_{0}$ such that $\left\|D_{k} \nabla f\left(z_{k}\right)\right\| \geqslant \epsilon_{0}$. By Lemma 1 , we have

$$
\begin{aligned}
\left|r_{k}-1\right| & \leqslant \frac{\frac{1}{2} c\left\|d_{k}\right\|^{2}-o\left(\left\|d_{k}\right\|\right)}{\left|q_{k}(0)-q_{k}\left(d_{k}\right)\right|} \\
& \leqslant \frac{\frac{1}{2} c\left\|d_{k}\right\|^{2}-o\left(\left\|d_{k}\right\|\right)}{\frac{1}{2} \alpha \epsilon_{0} \min \left\{\Delta_{k}, 1, \frac{\epsilon_{0}}{c}\right\}} \\
& \leqslant \frac{\frac{1}{2} c\left(\Delta_{k}\right)^{2}-o\left(\left(\Delta_{k}\right)\right)}{\frac{1}{2} \alpha \epsilon_{0} \min \left\{\Delta_{k}, 1, \frac{\epsilon_{0}}{c}\right\}}
\end{aligned}
$$

and $\left|r_{k}-1\right| \rightarrow 0$ as $\Delta_{k} \rightarrow 0$. By the monotone descent property, continuity, and the boundedness of $f\left(z_{k}\right)$ on $L\left(z_{0}\right)$, we obtain

$$
\lim _{k \rightarrow \infty} \operatorname{Ared}\left(d_{k}\right)=0, \lim _{k \rightarrow \infty} \operatorname{Pred}\left(d_{k}\right)=0 .
$$

However, by (16) and Method 1, we know that $r_{k} \geqslant \eta_{2}$ holds for $k$ sufficiently large and $\Delta_{k+1} \geqslant \Delta_{k}$. Thus there exists $\Delta>0$ and $\gamma>0$ that $\Delta_{k} \geqslant \gamma \tilde{\Delta}>0$ hold for any $k$ satisfying $\Delta_{k} \leqslant \tilde{\Delta}$, and obtain

$$
\operatorname{Pred}\left(d_{k}\right) \geqslant \frac{1}{2} \alpha \epsilon_{0} \min \left\{\Delta_{k}, 1, \frac{\epsilon_{0}}{c}\right\}>0 .
$$

Clearly, (17) and (18) are contradictory, therefore,

$$
\liminf _{k \rightarrow \infty}\left\|\nabla f\left(z_{k}\right)\right\|=0 .
$$

Theorem 2 Let $\left\{z_{k}\right\}$ be a sequence generated by Method 1. If $z^{*}$, an accumulation point of $\left\{z_{k}\right\}$, is a solution of problem (8) and any $V_{k} \in \partial_{C} H\left(z^{*}\right)$ has full rank. Then we know that $\left\{z_{k}\right\}$ is Q-quadratic convergent.

Proof: By Definition 3, $H$ is strongly semi-smooth, then it is locally Lipschitzian. Since $\mu_{k}=\mu\left\|f\left(z_{k}\right)\right\|^{2}$, $\mu \in(0,1)$, then $\limsup _{k \rightarrow \infty} \mu_{k} / O\left(\left\|H\left(z_{k}\right)\right\|\right)<\infty$ and $\mu_{k}=O\left(\left\|H\left(z_{k}\right)\right\|\right)$. The proof is then complete by following the similar proof of Theorem 5.3 in Ref. 29.

\section{NUMERICAL RESULTS}

In this section, Method 1 is applied to two general stochastic linear complementarity problems (Examples 1 and 2) and a refinery production problem ${ }^{8,33}$ (Example 3). The codes are written using MATLAB 7.0 and the parameters are taken as $\eta_{1}=10^{-5}, \eta_{2}=$ $0.75, \gamma_{1}=0.5, \gamma_{2}=2.0, \Delta_{\max }=10^{10}, \rho=0.99$, and $\mu=0.5$ with stopping rule $\left\|D_{k} g_{k}\right\| \leqslant 10^{-6}$ or $k_{\max }=50000$.

In the numerical experiments, the initial points are given randomly. We use $n$ to denote the dimension of the problem, and $x^{*}$ the minimum point of $f(z)$.

Example 1 Consider problem (1) with

$$
\begin{aligned}
& A_{1}(\omega)=\left(\begin{array}{cc}
-\frac{3}{2}+\omega & 2 \\
0 & -\frac{3}{2}+\omega
\end{array}\right), b_{1}(\omega)=\left(\begin{array}{l}
-\frac{3}{2}+\omega \\
-\frac{3}{2}+\omega
\end{array}\right), \\
& A_{2}(\omega)=\left(\begin{array}{cc}
\frac{5}{2}+\omega & 2 \\
0 & \frac{5}{2}+\omega
\end{array}\right), b_{2}(\omega)=\left(\begin{array}{l}
-\frac{3}{2}+\omega \\
-\frac{3}{2}+\omega
\end{array}\right), \\
& \Omega=\{0,1\}, p_{i}=P\left(\omega_{i} \in \Omega\right)=0.5, i=1,2 \text {, and } \lambda=1 . \\
& \text { The results are shown in Table } 1 .
\end{aligned}
$$


Table 1 Numerical results of Example 1.

\begin{tabular}{cc}
\hline$x^{*}$ & $f\left(z^{*}\right)$ \\
\hline$(0.3517,-0.5)$ & $4.4691 \times 10^{-16}$ \\
$(0.3681,-0.5)$ & $2.0791 \times 10^{-19}$ \\
$(0.4063,-0.5)$ & $7.1033 \times 10^{-18}$ \\
$(0.4752,-0.5)$ & $1.3102 \times 10^{-12}$ \\
$(0.3744,-0.5)$ & $3.9822 \times 10^{-13}$ \\
$(0.4023,-0.5)$ & $9.3473 \times 10^{-19}$ \\
$(0.4422,-0.5)$ & $3.7104 \times 10^{-14}$ \\
$(0.4814,-0.5)$ & $7.2941 \times 10^{-19}$ \\
$(0.3685,-0.5)$ & $1.1621 \times 10^{-12}$ \\
$(0.2154,-0.5)$ & $2.8290 \times 10^{-19}$ \\
\hline
\end{tabular}

Example 2 Consider problem (1) with

$$
\begin{aligned}
A(\omega) & =\left(\begin{array}{cccc}
\frac{1}{2}+\omega & 2 & \cdots & 2 \\
0 & \frac{1}{2}+\omega & \cdots & 2 \\
\vdots & \vdots & \ddots & \vdots \\
0 & 0 & \cdots & \frac{1}{2}+\omega
\end{array}\right)_{n \times n}, \\
b(\omega) & =\left(\begin{array}{c}
-\frac{3}{2}+\omega \\
\vdots \\
-\frac{3}{2}+\omega
\end{array}\right)_{n \times 1},
\end{aligned}
$$

$\Omega=\{0,1\}, p_{i}=P\left(\omega_{i} \in \Omega\right)=0.5, i=1,2$, and $\lambda=1$.

In this example, we select different dimensions $n$. The problems numerical results corresponding to different $n$ are given in Table 2, where the numerical experiments were carried out 10 times for each $n$.

The values of $f$ are degressive rapidly showing that the Method 1 is effective for solving Examples 1 and 2, see Fig. 1. The problem is complex when $n=800$ in Example 2, which has $x^{\prime}, x^{\prime \prime} \in \mathbb{R}^{800}$, $y_{i} \in \mathbb{R}^{800}, i=1, \ldots, 1600$, and $z \in \mathbb{R}^{1281600}$. The numerical results of Example 2 show that Method 1 is effective in solving this kind of general stochastic linear complementarity problems.

Example 3 Consider the refinery production problem $^{8,33}$, where $A_{1}(\omega)$ is an identity matrix, $b_{1}(\omega)=$ 0 ,

$$
\begin{aligned}
A_{2}(\omega) & =\left(\begin{array}{ccccc}
0 & 0 & 1 & -2-\omega_{1} & -3 \\
0 & 0 & 1 & -6 & \omega_{2}-3.4 \\
-1 & -1 & 0 & 0 & 0 \\
2+\omega_{1} & 6 & 0 & -\omega_{3} & -\omega_{3} \\
3 & 3.4-\omega_{2} & 0 & -\omega_{4} & \omega_{4}
\end{array}\right), \\
b_{2}(\omega) & =\left(\begin{array}{c}
-2 \\
-3 \\
-100 \\
180+\omega_{3} \\
162+\omega_{4}
\end{array}\right),
\end{aligned}
$$

$\lambda=0, k=10^{i}, i=2,3,4,2 x_{1}+3 x_{2}$ is the initial production cost, and $\omega_{j}$ satisfy the distribution

$$
\begin{aligned}
& \omega_{1} \approx u[-0.8,0.8], \\
& \omega_{2} \approx \mathrm{e}(\lambda=2.5), \\
& \omega_{3} \approx N(0,12), \\
& \omega_{4} \approx N(0,9) .
\end{aligned}
$$

Generated samples $\omega_{j}^{k}, j=1, \ldots, 4, k=1,2, \ldots, K$, from their respective $99 \%$ confidence intervals are, except for uniform distributions,

$$
\begin{aligned}
& \omega_{1}^{k} \in I_{1}=[-0.8,0.8], \\
& \omega_{2}^{k} \in I_{2}=[0.0,1.84], \\
& \omega_{3}^{k} \in I_{3}=[-30.91,30.91], \\
& \omega_{4}^{k} \in I_{4}=[-23.18,23.18] .
\end{aligned}
$$

For each $(j, i)$, the mean of the samples $\omega_{j}^{k}$ that belong to the subinterval $I_{j, i}$ is $v_{j, i}$ and the estimate probability of $v_{j, i}$ is $p_{j, i}=k_{j, i} / K$, where $k_{j, i}$ is the number of samples $\omega_{j}^{k} \in I_{j, i}$. Denote $N=m_{1} \times$ $m_{2} \times m_{3} \times m_{4}$ and the joint distribution $\left\{\left(\omega^{l}, p^{l}\right) \mid\right.$ $l=1,2, \ldots, N\}$ as

$$
\omega^{l}=\left(\begin{array}{l}
v_{1, i_{1}} \\
v_{2, i_{2}} \\
v_{3, i_{3}} \\
v_{4, i_{4}}
\end{array}\right), \quad p^{l}=p_{1, i_{1}} p_{2, i_{2}} p_{3, i_{3}} p_{4, i_{4}}
$$

for $i_{1}=1, \ldots, m_{1}, i_{2}=1, \ldots, m_{2}, i_{3}=1, \ldots, m_{3}$, $i_{4}=1, \ldots, m_{4}$. The conditions are assumed for two cases; case 1: $m_{1}=0, m_{2}=0, m_{3}=15, m_{4}=15$, case 2: $m_{1}=5, m_{2}=9, m_{3}=7, m_{4}=11$.

Tables 3 and 4 show that the minimization of $f$ and the minimum product cost $2 x_{1}^{k}+3 x_{2}^{k}$ are all computed effectively by Method 1 . The numerical results sufficiently show that the projected trust region method is a reliable solver for the proposed general stochastic complementarity problems.

\section{CONCLUSIONS}

In this study, a kind of general stochastic complementarity problem is considered and solved by a projected trust region method. This kind of general stochastic complementarity problem is more general and contains many models of practical problems, such as the refinery production problem. The global convergence of the projected trust region method is proved under general conditions, which is also verified by some numerical results. 
Table 2 Numerical results of Example 2.

\begin{tabular}{ccccc}
\hline \multicolumn{5}{c}{$f\left(z^{*}\right)$} \\
\\
\hline$n=10$ & $n=100$ & $n=200$ & $n=300$ & $n=800$ \\
\hline $5.32 \times 10^{-13}$ & $2.22 \times 10^{-12}$ & $5.69 \times 10^{-11}$ & $1.77 \times 10^{-7}$ & $3.43 \times 10^{-5}$ \\
$3.49 \times 10^{-19}$ & $2.32 \times 10^{-11}$ & $7.53 \times 10^{-12}$ & $8.52 \times 10^{-8}$ & $2.40 \times 10^{-6}$ \\
$2.75 \times 10^{-15}$ & $5.84 \times 10^{-12}$ & $9.22 \times 10^{-12}$ & $2.35 \times 10^{-7}$ & $2.38 \times 10^{-5}$ \\
$2.25 \times 10^{-19}$ & $2.00 \times 10^{-15}$ & $8.76 \times 10^{-12}$ & $1.77 \times 10^{-7}$ & $7.20 \times 10^{-5}$ \\
$5.43 \times 10^{-19}$ & $7.19 \times 10^{-12}$ & $4.02 \times 10^{-11}$ & $5.32 \times 10^{-7}$ & $5.39 \times 10^{-6}$ \\
$1.72 \times 10^{-13}$ & $6.88 \times 10^{-11}$ & $7.54 \times 10^{-12}$ & $4.02 \times 10^{-7}$ & $4.80 \times 10^{-7}$ \\
$1.17 \times 10^{-18}$ & $9.13 \times 10^{-12}$ & $3.22 \times 10^{-11}$ & $4.02 \times 10^{-7}$ & $3.91 \times 10^{-5}$ \\
$2.17 \times 10^{-16}$ & $2.66 \times 10^{-12}$ & $4.01 \times 10^{-13}$ & $3.23 \times 10^{-9}$ & $1.03 \times 10^{-5}$ \\
$3.18 \times 10^{-13}$ & $2.37 \times 10^{-12}$ & $2.06 \times 10^{-11}$ & $4.03 \times 10^{-7}$ & $2.07 \times 10^{-6}$ \\
$2.50 \times 10^{-13}$ & $4.02 \times 10^{-13}$ & $5.07 \times 10^{-11}$ & $4.01 \times 10^{-7}$ & $3.76 \times 10^{-5}$ \\
\hline
\end{tabular}

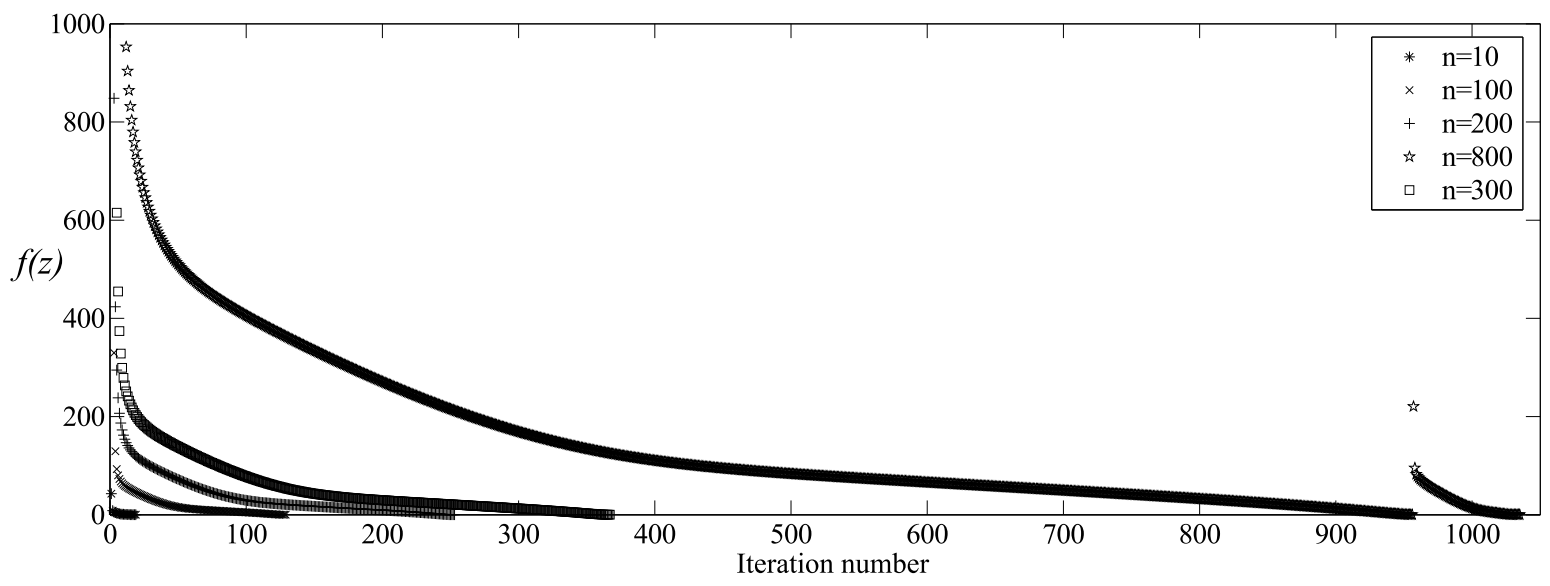

Fig. 1 The values of $f(z)$.

Table 3 Numerical results of Example 3, case 1.

\begin{tabular}{lccc}
\hline$k$ & $x^{*}$ & $f\left(z^{*}\right)$ & $2 x_{1}^{k}+3 x_{2}^{k}$ \\
\hline $10^{2}$ & $(34.97,19.03,0,0.24,0.51)$ & 1.10 & 127.03 \\
$10^{2}$ & $(34.96,19.04,0,0.24,0.51)$ & 1.10 & 127.03 \\
$10^{3}$ & $(34.97,19.03,0,0.24,0.51)$ & 1.10 & 127.03 \\
$10^{3}$ & $(34.96,19.04,0,0.24,0.51)$ & 1.10 & 127.04 \\
$10^{4}$ & $(35.32,19.09,0,0.26,0.46)$ & 1.48 & 127.90 \\
$10^{4}$ & $(35.32,19.09,0,0.26,0.46)$ & 1.48 & 127.91 \\
\hline
\end{tabular}

Table 4 Numerical results of Example 3, case 2.

\begin{tabular}{lccc}
\hline$k$ & $x^{*}$ & $f\left(z^{*}\right)$ & $2 x_{1}^{k}+3 x_{2}^{k}$ \\
\hline $10^{2}$ & $(35.11,19.99,0,0.34,0.51)$ & 1.36 & 130.19 \\
$10^{2}$ & $(35.12,18.88,0,0.25,0.50)$ & 1.36 & 126.88 \\
$10^{3}$ & $(35.12,18.88,0,0.25,0.50)$ & 1.36 & 126.88 \\
$10^{3}$ & $(35.12,18.88,0,0.25,0.50)$ & 1.36 & 126.88 \\
$10^{4}$ & $(34.91,19.09,0,0.25,0.50)$ & 1.36 & 127.09 \\
$10^{4}$ & $(34.91,19.09,0,0.25,0.50)$ & 1.36 & 127.09 \\
\hline
\end{tabular}

Acknowledgements: This work was supported by the National Natural Science Foundation of China (No. 11671220) and the Shandong Provincial Nature Science Foundation, China (No. ZR2016AM29). The authors thank the anonymous referees for their numerous insightful comments and suggestions, which have greatly improved this paper.

\section{REFERENCES}

1. Bakshi G, Cao C, Chen ZW (1997) Empirical performance of alternative option pricing models. $J$ Finance 52, 2003-49.

2. Black F, Scholes M (1973) The pricing of options and corporate liabilities. $J$ Polit Econ 81, 637-54.

3. Zhang YP (2013) An American option pricing model with historical price constraints based on linear complementarity. MSc thesis, Dalian Univ of Technology. [in Chinese]

4. Hamatani K, Fukushima M (2011) Pricing American options with uncertain volatility through stochastic linear complementarity models. Comput Optim Appl 50, 263-86. 
5. Huang J, Pang JS (1998) Option pricing and linear complementarity. $J$ Comput Finance 2, 31-60.

6. Insley M, Rollins K (2005) On solving the multirotational timber harvesting problem with stochastic prices: A linear complementarity formulation. Am J Agric Econ 87, 735-55.

7. Sun HC (2009) A quadratically convergent algorithm for generalized linear complementarity problem on supply chain equilibrium model. In: 2009 International Symposium on Intelligent Ubiquitous Computing and Education, Chengdu, China, pp 230-3.

8. Chen XJ, Fukushima M (2005) Expected residual minimization method for stochastic linear complementary problems. Math Oper Res 30, 785-1040.

9. Liu ZM, Du SQ, Wang RY (2016) A new conjugate gradient projection method for solving stochastic generalized linear complementarity problems. J Appl Math Phys 4, 1024-31.

10. Zhou GL, Caccetta L (2008) Feasible semismooth Newton method for a class of stochastic linear complementarity problems. J Optim Theory Appl 139, 379-92.

11. Chen XJ, Zhang C, Fukushima M (2009) Robust solution of monotone stochastic linear complementarity problems. Math Program 117, 51-80.

12. Gürkan G, Özge AY, Robinson SM (1999) Samplepath solution of stochastic variational inequalities. Math Program 84, 313-33.

13. Han JY, Xiu NH, Qi HD (2006) Nonlinear Complementary Theory and Algorithm, Shanghai Scientific and Technical Publishers. [in Chinese]

14. Caccetta L, Qu B, Zhou GL (2011) A globally and quadratically convergent method for absolute value equations. Comput Optim Appl 48, 45-58.

15. Mangasarian OL (2007) Absolute value programming. Comput Optim Appl 36, 43-53.

16. Cottle RW, Pang JS, Stone RE (1992) Linear Complementarity Problem, Academic Press, New York.

17. Facchinei F, Pang JS (2003) Finite-Dimensional Variational Inequalities and Complementarity Problems, Springer, New York.

18. Chen JS (2007) On some NCP-functions based on the generalized Fischer-Burmeister function. Asia-Pac $J$ Oper Res 24, 401-20.

19. Chen JS, Huang ZH, She CY (2011) A new class of penalized NCP-functions and its properties. Comput Optim Appl 50, 49-73.

20. Fischer A (1992) A special Newton-type optimization method. Optimization 24, 269-84.

21. Dembo RS, Eisenstat SC, Steihaug T (1982) Inexact Newton method. SIAM J Numer Anal 19, 400-8.

22. Gabriel SA, Pang JS (1994) A trust-region method for constrained nonsmooth equations. In: Hager WW, Hearn DW, Pardalos PM (eds), Large Scale Optimization, Springer, Boston, MA, pp 155-81.

23. Fan B (2017) A nonmonotone Levenberg-Marquardt method for nonlinear complementarity problems under local error bound. Comput Appl Math 36, 185-202.

24. Kanzow C, Yamashita N, Fukushima M (2004) Levenberg-Marquardt methods with strong local convergence properties for solving nonlinear equations with convex constrains. J Comput Appl Math 172, 375-97.

25. Jiang HY, Fukushima M, Qi LQ, Sun DF (1998) A trust-region method for solving generalized complementarity problems. SIAM J Optim 8, 140-57.

26. Sagara N, Fukushima M (2005) Trust region method for nonsmooth convex optimization. $J$ Ind Manag Optim 1, 171-80.

27. Ling C, Qi LQ, Zhou GL, Caccetta L (2008) The $S C^{1}$ property of an expected residual function arising from stochastic complementarity problems. Oper Res Lett 36, 456-60.

28. Coleman TF, Li YY (1996) An interior trust region approach for nonlinear minimization subject to bounds. SIAM J Optim 6, 418-45.

29. Kanzow C, Petra S (2007) Projected filter trust region method for a semismooth least squares formulation of mixed complementarity problems. Optim Methods Softw 22, 713-35.

30. Kanzow C, Klug A (2006) On affine-scaling interiorpoint Newton methods for nonlinear minimization with bound constraints. Comput Optim Appl 35, 177-97.

31. Ma CF (2010) Optimization Method and its Matlab Programming, Science Press, Beijing. [In Chinese]

32. Clarke F (1990) Optimization and Nonsmooth Analysis, Classics in Applied Mathematics, SIAM, Philadelphia.

33. Kall P, Wallace S (1994) Stochastic Programming, John Wiley \& Sons Inc., New York. 